\title{
Can a New Algorithm of Cardiac Resynchronization Therapy (Adaptive CRT) Expand Its Utility?
}

\author{
Takashi Kurita, MD, PhD
}

C ardiac resynchronization therapy (CRT) is an established essential strategy to manage patients with moderate to severe heart failure $(\mathrm{HF}) .^{1-3}$ However, the obvious favorable effects of CRT in many randomized studies have been limited to patients with complete left bundle branch block (CLBBB) and a QRS width $\geq 150$ ms. $^{1-3}$

Even in patients with severely wide QRS, several clinical studies have shown that CRT is least effective in those with complete right bundle branch block (CRBBB) and those with non-specific intraventricular conduction delay as compared with CLBBB patients.,5 Furthermore, the EchoCRT study demonstrated that CRT is not effective, and even harmful, for reducing HF hospitalizations and deaths of patients with a narrow QRS $<130 \mathrm{~ms}$ and echocardiographic desynchronization. ${ }^{6}$ According to the results from the EchoCRT study, the ESC 2016 guidelines classified such patients as a Class III indication for CRT. ${ }^{7}$ On the other hand, a subanalysis of the EchoCRT study showed

\section{Article p 18}

that CRT is not always harmful in the subgroup with a relatively small left ventricular volume. ${ }^{8}$ Based on this subanalysis, the lower limit of CRT indications in terms of QRS width was determined as $120 \mathrm{~ms}$ in the $2018 \mathrm{JCS} /$ JHRS guidelines. ${ }^{9}$

Therefore, demonstrating CRT efficacy in patients with a moderately wide $\mathrm{QRS}$ or non-CLBBB QRS morphology is the next challenging frontier of this therapy, and much attention has been focused on whether the problem can be resolved by advanced capability of CRT or whether it is a fundamental limitation of CRT.

Under these circumstances, adaptive CRT (aCRT) has been introduced with great expectation that it will resolve some of the problems of CRT. The aCRT is a novel algorithm that periodically measures intrinsic atrioventricular (AV) conduction and dynamically adjusts CRT pacing

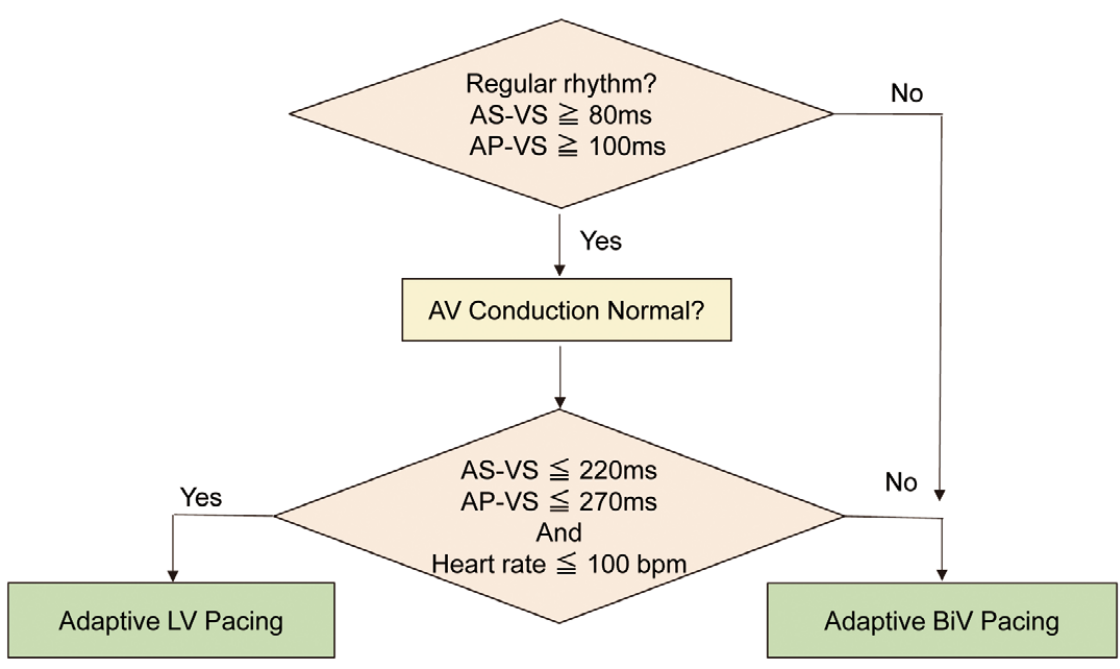

Figure. Algorithm of adaptive cardiac resynchronization therapy (aCRT). AP-VS, atrial pacing-ventricular sensing; AS-VS, atrial sensing-ventricular sensing; AV, atrioventricular; BiV, biventricular; bpm, beats/min; LV, left ventricular.

The opinions expressed in this article are not necessarily those of the editors or of the Japanese Circulation Society.

Received November 5, 2019; accepted November 5, 2019; J-STAGE Advance Publication released online December 4, 2019

Division of Cardiovascular Center, Kindai University, Osaka-Sayama, Japan

Mailing address: Takashi Kurita, MD, PhD, Division of Cardiovascular Center, Kindai University, 377-2 Ohono-Higashi, Osaka-

Sayama 589-8511, Japan. E-mail: kuritat@med.kindai.ac.jp

ISSN-1346-9843 All rights are reserved to the Japanese Circulation Society. For permissions, please e-mail: cj@j-circ.or.jp 
conditions as necessary to maximize the effects of CRT. This algorithm also provides RV-synchronized LV pacing when AV conduction is normal and biventricular (BiV) pacing when AV conduction is prolonged (Figure).

This intriguing function has been evaluated by the Adaptive CRT study, ${ }^{10}$ a prospective, multicenter, randomized, double-blind clinical trial that compared CRT with the aCRT algorithm settings with conventional CRT with AV and VV settings optimized by a standardized echocardiographic protocol.

The main study and several subanalyses of the Adaptive CRT study found the following:

- aCRT was safe and at least as effective as conventional CRT with comprehensive echocardiographic optimization $^{10}$

- RV pacing was reduced by $44 \%$ in the aCRT arm ${ }^{11}$

- A higher percentage of synchronized LV pacing $(\geq 50 \%)$ was independently associated with decreased risk of death or HF hospitalization ${ }^{11}$

- aCRT reduced the risk of AF compared with those receiving conventional $\mathrm{CRT}$ in the subgroups with prolonged AV conduction at baseline ${ }^{\mathbf{1 2}}$

- aCRT was associated with improved patient outcomes over echo-optimized CRT in patients with preserved AV conduction, LBBB, and moderately wide QRS of $120-150 \mathrm{~ms}^{13}$

The last point was reported by Yamasaki et al, ${ }^{13}$ using a clinical composite score including factors such as death, HF hospitalization, crossover from an assigned randomization, the NYHA class, and patient global assessment that categorizes patients as improved. Therefore, the clinical implication is rather indistinct.

A corresponding study by Ueda et $\mathrm{al}^{\mathbf{1 4}}$ was designed to examine the clinical benefit of the aCRT algorithm in patients with mildly wide QRS (120-150 ms) or non-LBBB morphology. In this issue of the Journal, they demonstrate that aCRT significantly improved the composite clinical outcome of just 2 main endpoints (cardiac death and/or HF hospitalization). However, to promote use of aCRT with confidence in patients with a moderately wide QRS and/or non-CLBBB, several problems remain to be resolved. First, this study was retrospective with a small number of patients. Although the number of patients in each group was accidentally almost even, and much of the clinical background happened to be similar between the 2 groups, there might have been a concealed or unknown bias. Ideally, a prospective, large, randomized study, principally targeting patients with a moderately wide QRS and/or non-CLBBB is needed to obtain further strong evidence. Second, the authors do not explain why the primary endpoint decreased in the aCRT group despite the percentage of responders (defined as an improvement in the LVEF of $\geq 10 \%$ and/or reduction in the LVESV of $\geq 15 \%$ ) being comparable between the 2 groups (63\% [aCRT group] vs. 59\% [nonaCRT group], $\mathrm{P}=0.42$ ). Third, the mechanism of the beneficial effect of the aCRT in these particular patients is still unclear. The authors speculate that the aCRT algorithm, which reduces unnecessary RV pacing, preserves a positive net effect of CRT and results in a better clinical outcome. However, their study did not show what percentage of RV pacing was avoided in the aCRT group. Further- more, their speculation could not explain the beneficial effects of aCRT in patients with a wide and non-LBBB QRS.

Although the study by Ueda et al has several limitations, it shows us the expanding possibilities of CRT.

\section{Disclosures}

Takashi Kurita serves as a consultant to Japan Medtronic.

\section{References}

1. Cleland JGF, Daubert JC, Erdmann E, Freemantle N, Gras D, Kappenberger L, et al; for the Cardiac Resynchronisation-Heart Failure (CARE-HF) Study Investigators. The effect of cardiac resynchronization on morbidity and mortality in heart failure. $N$ Engl J Med 2005; 352: 1539-1549.

2. Tang AS, Wells GA, Talajic M, Arnold MO, Sheldon R, Connolly $\mathrm{S}$, et al; Resynchronization-Defibrillation for Ambulatory Heart Failure Trial Investigators. Cardiac-resynchronization therapy for mild-to-moderate HF. N Engl J Med 2010; 363: 2385-2395.

3. Moss AJ, Schuger C, Beck CA, Brown MW, Cannom DS, Daubert JP, et al. Reduction in inappropriate therapy and mortality through ICD programming. $N$ Engl J Med 2012; 367: $2275-2283$.

4. Bilchick KC, Kamath S, DiMarco JP, Stukenborg GJ. Bundlebranch block morphology and other predictors of outcome after cardiac resynchronization therapy in medicare patients. Circulation 2010; 122: 2022-2030.

5. Auricchio A, Prinzen FW. Non-responders to cardiac resynchronization therapy: The magnitude of the problem and the issues. Circ J 2011; 75: 521-527.

6. Ruschitzka F, Abraham WT, Singh JP, Bax JJ, Borer MS, Brugada J, et al; EchoCRT Study Group. Cardiac-resynchronization therapy in HF with a narrow QRS complex. N Engl J Med 2013; 369: $1395-1405$.

7. Ponikowski P, Voors AA, Anker SD, Bueno H, Cleland JGF, Coats AJS, et al; ESC Scientific Document Group. 2016 ESC Guidelines for the diagnosis and treatment of acute and chronic HF: The Task Force for the diagnosis and treatment of acute and chronic heart failure of the European Society of Cardiology (ESC) developed with the special contribution of the Heart Failure Association (HFA) of the ESC. Eur Heart J 2016; 37: 2129-2200.

8. Varma N, Sogaard P, Bax JJ, Abraham WT, Borer JS, Dickstein $\mathrm{K}$, et al. Interaction of left ventricular size and sex on outcome of cardiac resynchronization therapy among patients with a narrow QRS duration in the EchoCRT Trial. J Am Heart Assoc, doi:10.1161/JAHA.118.009592.

9. Kurita T, Nogami A. 2018 JCS/JHRS guideline on non-pharmacotherapy of cardiac arrhythmias. http://www.j-circ.or.jp/guideline/ pdf/JCS2018_kurita_nogami.pdf (accessed August 26, 2019).

10. Birnie D, Lemke B, Ánuma K, Krum H, Lee KL, Gasparini M, et al. Clinical outcomes with synchronized left ventricular pacing: Analysis of the adaptive CRT trial. Heart Rhythm 2013; 10: $1368-1374$.

11. Martin DO, Lemke B, Birnie D, Krum H, Lee KL, Aonuma K, et al; Adaptive CRT Study Investigators. Investigation of a novel algorithm for synchronized left-ventricular pacing and ambulatory optimization of cardiac resynchronization therapy: Results of the adaptive CRT trial. Heart Rhythm 2012; 911: 1807-1814.

12. Birnie D, Hudnall H, Lemke B, Aonuma K, Lee KL, Gasparini $\mathrm{M}$, et al. Continuous optimization of cardiac resynchronization therapy reduces atrial fibrillation in heart failure patients: Results of the Adaptive Cardiac Resynchronization Therapy Trial. Heart Rhythm 2017; 14: 1820-1825.

13. Yamasaki H, Lustgarten D, Cerkvenik J, Birnie D, Gasparini M, Lee KL, et al. Adaptive CRT in patients with normal AV conduction and left bundle branch block: Does QRS duration matter? Int J Cardiol 2017; 240: 297-301.

14. Ueda N, Noda T, Ishibashi K, Nakajima K, Kataoka N, Kamakura $\mathrm{T}$, et al. Efficacy of a device-based continuous optimization algorithm for patients with cardiac resynchronization therapy. Circ J 2020; 84: 18-25. 